\title{
Effects of shRNA Expression Vector-mediated Glutathione S-transferase P1 Gene Silencing on Prostate Cancer DU145 Cells
}

\author{
Ruirui Xu ${ }^{1, *}$, Zhufu Shao ${ }^{2}$, Zhi Xiao" \\ ${ }^{1}$ Department of Nephrology, Binzhou People's Hospital, Binzhou City, P. R. China \\ ${ }^{2}$ Department of Critical Care Medicine, Binzhou People's Hospital, Binzhou City, P. R. China \\ Email address: \\ RuiruiXu2019@163.com (Ruirui Xu) \\ *Corresponding author
}

To cite this article:

Ruirui Xu, Zhufu Shao, Zhi Xiao. Effects of shRNA Expression Vector-mediated Glutathione S-transferase P1 Gene Silencing on Prostate Cancer DU145 Cells. American Journal of Clinical and Experimental Medicine. Vol. 7, No. 5, 2019, pp. 119-125. doi: 10.11648/j.ajcem.20190705.13

Received: September 23, 2019; Accepted: October 31, 2019; Published: November 18, 2019

\begin{abstract}
Objective: This study aims to explore the impact of silent GSTP1 gene on the proliferation activity, cell cycle and apoptotic rate of the androgen-independent prostate cancer cell strain DU145. Methods: Thirty subjects with benign prostatic hyperplasia (BPH) who had been subject to urinary surgical resection and pathological diagnosis, 30 subjects with radically cured prostate cancer (PCa), and another 30 subjects with hormone refractory prostate cancer (HRPC) were enrolled. The GSTP1 expression in the subjects was tested with the immunohistochemical S-P method, the GSTP1 expression in the blood serum of the patients with prostatic hyperplasia and prostate cancer was tested with Elisa, and the mRNA level of the GSTP1 gene after the DU145 cells were transfected with shRNA was tested. Results: Four of the HRPC patients were complicated with bone metastasis, two with bladder invasion, and five with liver and lung metastasis. GSTP1 showed high expression in tissues with prostatic hyperplasia and hormone refractory prostate cancer and low expression in tissues with prostate cancer, and the differences were statistically significant $(\mathrm{P}<0.05)$. Difference was found among the serum concentrations of the BPH group, the PCa group and the HRPC group. The HRPC group showed serum GSTP1 concentration higher than that of the $\mathrm{PCa}$ group $(\mathrm{P}<0.01)$, the $\mathrm{BPH}$ group also showed serum GSTP1 concentration higher than that of the PCa group $(\mathrm{P}<0.01)$, and the difference between the serum concentrations of the BPH group and the HRPC group was not statistically significant ( $\mathrm{P}>0.05)$. The GSTP1 mRNA content decreased significantly after shRNA554 intervention, with an IOD value of 67.1 \pm 8.7 . The intervention effect was evidently lower than that of the carrier shRNA255 group $(162.2 \pm 12.6)$ and the shRNA593 group (114.5 \pm 10.6$)$ with statistically significant difference $(\mathrm{P}<0.01)$. Conclusion: The expression vector-mediated GSTP1-shRNA554 transfection can reduce the mRNA level of the GSTP1 gene of the androgen-independent prostate cancer DU145 cells and inhibit the proliferation activity of the in vitro cultivated DU145 cells.
\end{abstract}

Keywords: shRNA Expression Vector, Glutathione S-transferase P1, Gene Silencing, Prostate Cancer, DU145

\section{Introduction}

As the most common malignancy in the United States and Europe, prostate cancer ( $\mathrm{PCa}$ ) is now showing the second greatest morbidity and mortality in the males of Western countries. There are annually 28,900 people dying of HRPC in the United States. With the improvement of living standards and average life expectancy, prostate cancer is having an increasing morbidity in China, ranking the third among the malignant tumors of the male urinary and reproductive system [1]. The prostate cancers that recur and progress after continued endocrine therapy include androgen-independent prostate cancer (AIPC) and hormone-refractory prostate cancer (HRPC). Two thirds of the prostate cancer patients can only be treated with endocrine therapy featuring maximal androgen blockade (MAB) once the diagnosis shows that they have missed the opportunity for radical cure [2]. After 12 to 18 months, $70 \%$ to $80 \%$ of the patients would turn to be 
androgen-independent with recurrence or distant metastasis and an average survival less than one year [3]. There is currently no effective therapy for hormone-refractory prostate cancer, and a number of scholars both at home and abroad are focusing on the research and development of a new therapy for patients of this category.

Glutathione S-transferases (GSTs) are dimeric isoenzymes with strong catalytic effect to catalyze substrate molecules, electron affinity, and reduced glutathione conjugation. They can promote metabolism and inactivation, and are one of the most important phase II metabolic enzymes for the in vivo biotransformation [4]. The GST superfamily members can be divided into four major subfamilies $(\alpha, \mu, \pi$ and $\theta)$ according to their chromosome locations and sequence homology. GSTP1 gene encoded protein GST- $\pi$ has become a hot topic of research in recent years due to its close relationship with the occurrence and development of malignant tumors [5]. It has been reported in gastrointestinal cancer studies that drug tolerance of cancers can be reduced by inhibiting the GSTP1 activity which can be mainly achieved by using signaling pathway drugs to inhibit its expression and using RNA interference to reduce its level of expression [6]. The relationship between GSTP1 and prostate cancer has been often reported in recent years [7].

With GSTP1 as the target of the gene therapy, this study uses short hairpin RNA (shRNA)-mediated RNA to interfere the silence GSTP1 gene to explore its impact on the proliferation activity, cell cycle and apoptotic rate of the androgen-independent prostate cancer cell strain DU145. Chemotherapeutic changes before and after the transfection are also observed.

\section{Materials and Methods}

\subsection{Materials}

The materials and apparatus used herein included: goat anti-mouse/rabbit antibodies, Beijing Kangpu Huiwei Technology Co., Ltd.; GSTP1 and ELISA kit containing chromogenic substrate 3,3',5,5'-tetramethylbenzidine (TMB), Amresco (US); androgen-independent prostate cancer cell strain DU145, Shanghai Ricky Biotechnology Co., Ltd.; PMIl640 culture medium, Beijing Ruizekang Biotechnology Co., Ltd.; Trizol Reagent and dNTP mixture, Amresco (US); shRNA, Chinese Academy of Medical Sciences \& Peking Union Medical College Institute of Biomedical Engineering; light microscope, Nikon (Japan); microplate reader, Shanghai AoXi Technology Instrument Co., Ltd.; IMT-2 inverted microscope, Olympus (Japan).

\subsection{Subjects}

30 subjects with benign prostatic hyperplasia (BPH) who had been subject to urinary surgical resection and pathological diagnosis, 30 subjects with radically cured prostate cancer (PCa), and another 30 subjects with hormone refractory prostate cancer (HRPC) were enrolled.

Inclusion criteria: (1) serum testosterone up to the castration level $(<50 \mathrm{ng} / \mathrm{m} 1)$; (2) three consecutive PSA increases in intervals of two weeks; (3) anti-androgen withdrawal treatment for more than 4 weeks; (4) PSA progression during the second-line endocrine therapy; and (5) progress in the bone or soft tissue metastasis.

The tissues were $10 \%$ formalin fixed, ordinary paraffin embedded, and prepared by HE staining. The tissues were observed with optical microscope for diagnosis. Typical tissue blocks confirmed with slice HE staining were selected and 5 $\mu \mathrm{m}$ thick white slices were excised from each of them for immunohistochemical staining. Five $\mathrm{ml}$ of peripheral blood was drawn from each subject for the determination of serum GSTP1 content by enzyme-linked immunosorbent assay (ELISA).

\subsection{Detection of GSTP1 Expression in Prostatic Hyperplasia and Prostate Cancer Tissues by Immunohistochemistry}

The prostate specimens were fixed with $5 \%$ formalin and stored at $-70^{\circ} \mathrm{C}$ before the surgical excision. The GSTP1 expression in the abovementioned specimen was tested with the immunohistochemical S-P method.

Criteria for GSTP1 staining positive expression: Yellow-brown cytoplasm or nucleus suggested positive staining. Otherwise, the staining was negative. The percentage of the positive cells was counted for three times and the average value was obtained. Percentage of positive cells $=$ number of positive cells / total number of cells $\times 100 \%$. Then the slices were divided into four levels according to the percentage of positive cells in each slice: negative (-, with percentage of positive cells lower than $1 \%$ ), weakly positive $(+$, with percentage of positive cells between $1 \%$ and $25 \%)$, moderately positive $(++$, with percentage of positive cells between $25 \%$ and $50 \%$ ) and strongly positive $(+++$, with percentage of positive cells greater than 50\%). Two pathologists would determine the results through blind slice reading with the help of image analysis system software. Criteria for determination: Five high-power fields $(\times 200)$ were randomly selected, and determination was done based on the sum of the scores for the staining intensity and positive cell percentage: 0 points for no staining, 1 point for weak staining (light yellow), 2 points for moderate staining (brown-yellow), and 3 points for strong staining (brown); 0 points for positive cell percentage $\leq 5 \%, 1$ point for $5 \%<$ positive cell percentage $\leq 25 \%, 2$ points for $25 \%<$ positive cell percentage $\leq 50 \%$, and 3 points for positive cell percentage $>$ $50 \%$. The two scores were summed, and 0 points stands for negative (-), 1-2 points stands for weakly positive (+), 3-4 points stands for moderately positive $(++)$, and 5-6 points stands for strongly positive $(+++)$ [8].

\subsection{Detection of GSTP1 Expression in Prostatic Hyperplasia and Prostate Cancer Serum by ELISA}

The blood serums of all the subjects were collected before surgery. The whole blood samples placed in the serum separator tube were put at room temperature for $1 \mathrm{~h}$ or $4^{\circ} \mathrm{C}$ over night. Then they were centrifuged at $1,000 \mathrm{rpm}$ for $10 \mathrm{~min}$, and the 
supernatant was taken for testing. The absorbance (also optical density, OD) was measured with a microplate reader at a $450 \mathrm{~nm}$ wavelength. The concentration of the sample was calculated, and the actual concentration should be the tested concentration multiplied by the sample dilution factor.

\section{5. shRNA Transfection and Screening}

The day before transfection, $1 \times 105$ DU145 cell strains were inoculated on 6-well plates, and $3 \mathrm{~mL}$ of basal medium containing FBS. The number of cells was selected for the initial inoculation, with cell confluency reaching $80 \%$ in $24 \mathrm{~h} .10 \mu \mathrm{l}$ of shRNA was diluted with $250 \mu \mathrm{l}$ of Opti-MEM I and mixed gently and evenly. After incubation for $30 \mathrm{~min}$, Lipofectamine 2000 was added, mixed gently, and incubated for $60 \mathrm{~min}$ at room temperature so as to allow the formation of complexes. The shRNA-Lipofectamine 2000 complexes were added to each well containing the cells and the medium. The complexes were mixed by gently shaking the culture plate back and forth. shRNA255, shRNA554, shRNA593 and Negative-shRNA were transfected separately and were inoculated to 96-well plates after $48 \mathrm{~h}$ using the limiting dilution assay.

Incubation was carried out at $37^{\circ} \mathrm{C}$ in the $\mathrm{CO}_{2}$ incubator for $48 \mathrm{~h}$, the cells were smeared after digestion with $0.5 \%$ trypsin digestion, and the number of transfected cells and their positive percentage were observed and calculated under the fluorescence microscope. The transfection efficiency levels of shRNA255, shRNA554 shRNA593 and Negative-shRNA were compared. Calculation of transfection efficiency: count the total number of the cells on the ordinary optical microscopic image and the number of fluorescent cells on the fluorescence microscopic image; transfection efficiency $=$ number of fluorescent cells / total number of cells $\times 100 \%$. The requirement for transfection intervention would have been met where the shRNA vector transfection efficiency was $80 \%$ or more.

\subsection{Detection of GSTP1 Gene mRNA Levels After Transfecting DU145 Cells with shRNA}

Add $1 \mathrm{ml}$ of Trizol reagent into each DU145 cell precipitate sample. Leave the homogenate sample to stand for $5 \mathrm{~min}$ before adding 0.5 times the volume of chloroform. Shaking for $1 \mathrm{~min}$ and centrifuge at $3,000 \mathrm{rpm}$ for $10 \mathrm{~min}$ at $4{ }^{\circ} \mathrm{C}$. Transfer the aqueous layer to a clean test tube, add 0.5 times the volume of isopropanol, and mix evenly. Leave to stand at $20^{\circ} \mathrm{C}$ for $30 \mathrm{~min}$, and centrifuge at $3,000 \mathrm{rpm}$ for $15 \mathrm{~min}$ at $4^{\circ} \mathrm{C}$. Remove the upper suspension and wash the RNA precipitate with $1 \mathrm{ml}$ of $75 \%$ ethanol. Mix evenly on a shaker, and then centrifuge at $73,000 \mathrm{rpm}$ for $5 \mathrm{~min}$ at $4{ }^{\circ} \mathrm{C}$ to discard the supernatant. Moderately dry the RNA precipitate, and add an appropriate amount of RNase-free water to fully dissolve the RNA. Centrifuge at 2,000 rpm for $5 \mathrm{~min}$. Primer sequences of GSTP1 gene: upstream primer 5'-CATCTACTCTCCCTACCAAC-3', downstream primer 5'-AGGATTTCAGCGTAGGAGAT-3'. Internal standard GAPDH: upstream primer 5'-GTCCATGACCCACACATCAC-3', downstream primer 5'-TGCTGTCACCCCACCTGTTA-3'. Conditions for PCR reactions: After evenly mixing the reactants, centrifuge at $3,000 \mathrm{rpm}$ for $10 \mathrm{~min}$, initial denaturation at $92^{\circ} \mathrm{C}$ for $60 \mathrm{~s}$, denaturation at $92^{\circ} \mathrm{C}$ for $45 \mathrm{~s}$, and anneal at $45^{\circ} \mathrm{C}$ for $30 \mathrm{~s}$ : extension at $70^{\circ} \mathrm{C}$ for $40 \mathrm{~s}$ with 30 cycles, and after the last cycle, extension at $72^{\circ} \mathrm{C}$ for $60 \mathrm{~s}$, and cessation reaction at $4^{\circ} \mathrm{C}$. The amplification products were subject to $3 \%$ agarose gel electrophoresis. The sample amount was $10 \mu \mathrm{l}$ and the voltage was $120 \mathrm{~V}$. Staining was performed with ethidium bromide (EB) after the electrophoresis, and the results were observed and photographed under UV lamps.

\subsection{Statistical Analysis}

All data were analyzed by SPSS 15.0. The measurement data were expressed as (mean \pm standard derivation) $(\mathrm{x} \pm \mathrm{s})$. All groups were subject to single-factor analysis of variance, and multiple groups were subject to analysis of variance. Two groups were compared by $\mathrm{t}$ test. $\mathrm{P}<0.05$ was considered statistically significant.

\section{Results}

\subsection{Clinical Pathological Characteristics of Subjects}

There were 30 subjects with pathologically diagnosed benign prostatic hyperplasia $(\mathrm{BPH}), 30$ subjects with radically cured prostate cancer $(\mathrm{PCa})$, and 30 subjects with hormone refractory prostate cancer (HRPC). All PCa patients were diagnosed by biopsy and reviewed by two pathologists. The age, clinical stages, Gleason scores and serum PSA are as follows in Table 1. Four of the HRPC patients were complicated with bone metastasis, two with bladder invasion, and five with liver and lung metastasis.

Table 1. Clinical pathological characteristics of included subjects.

\begin{tabular}{llll}
\hline & BPH & PCa & HRPC \\
\hline Age & $42.38 \pm 12.61$ & $41.82 \pm 11.97$ & $43.26 \pm 12.08$ \\
Stage pT2 & - & 18 & - \\
Stage pT3 & - & 12 & 12 \\
Stage pT4 & - & - & 18 \\
Gleason Score 2 3 & - & 8 & 4 \\
Gleason Score 4 6 & - & 14 & 16 \\
Gleason Score 7 10 & - & 8 & 10 \\
PSA (ng/ml) 0 4 & 24 & - & - \\
PSA (ng/ml) 5 10 & 6 & - & - \\
PSA (ng/ml) $11 \sim 20$ & - & 14 & - \\
PSA (ng/ml) $>20$ & - & 16 & 30 \\
\hline
\end{tabular}




\subsection{Detection of GSTP1 Expression in Prostatic Hyperplasia and Prostate Cancer Tissues by Immunohistochemistry}

Criteria for GSTP1 staining positive expression: Yellow-brown cytoplasm or nucleus suggested positive staining. Scores were obtained in accordance cell positive rate and the degree of staining. The two scores were summed, and 0 points stood for negative (-), 1-2 points stood for weakly positive $(+), 3-4$ points stood for moderately positive $(++)$, and 5-6 points stood for strongly positive $(+++)$ (Figure 1). GSTP1 showed high expression in tissues with prostatic hyperplasia and hormone refractory prostate cancer and low expression in tissues with prostate cancer, and the differences were statistically significant $(\mathrm{P}<0.05)$ (Table 2).
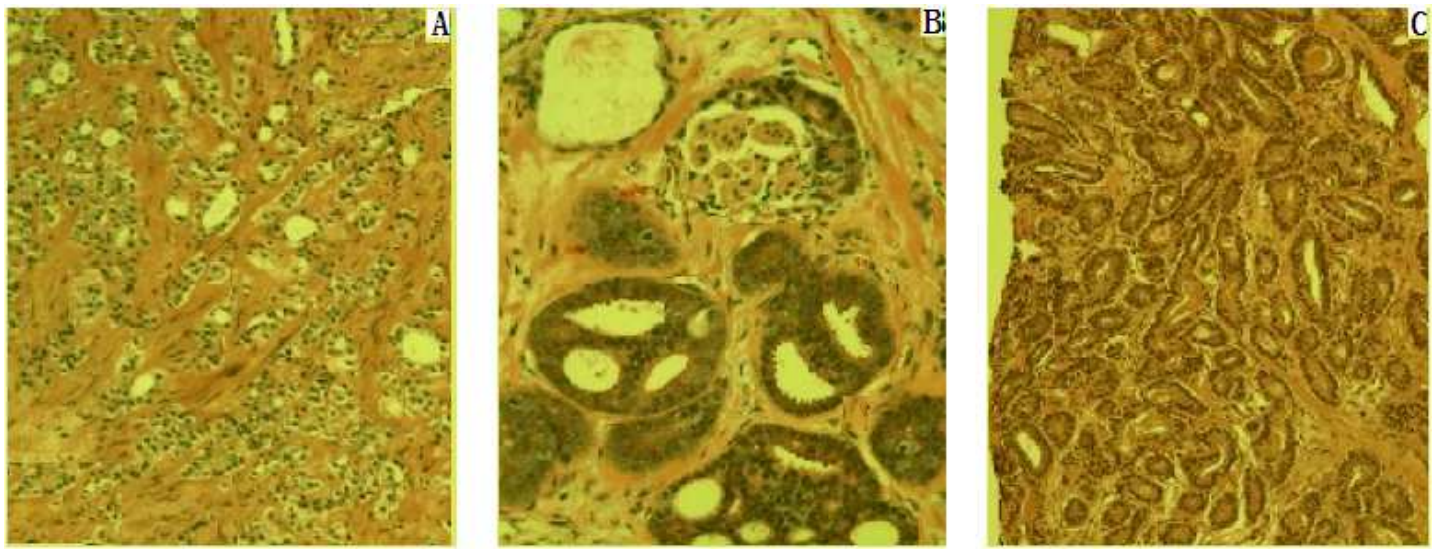

Figure 1. Detection of GSTP1 expression in prostatic hyperplasia and prostate cancer tissues by immunohistochemistry. A: Strong positive GSTP1 expression in prostatic hyperplasia tissues; B: GSTP1 expression in prostate cancer tissues before radical resection (negatively or mildly positively expressed in cancer glandular epithelium, and positively expressed in peripheral mesenchyme); $C$ : strong positive GSTP1 expression in hormone refractory prostate cancer tissues.

Table 2. Detection of GSTP1 expression in prostatic hyperplasia and prostate cancer tissues by immunohistochemistry.

\begin{tabular}{llllll}
\hline & \multicolumn{2}{l}{ GSTP1 expression } & & & \\
\cline { 2 - 5 } & - & + & ++ & +++ & Positive rate (\%) \\
\hline $\mathrm{BPH}$ & 0 & 4 & 8 & 18 & $100 \% \mathrm{a}$ \\
$\mathrm{PCa}$ & 20 & 5 & 5 & 0 & $33.33 \% \mathrm{~b}$ \\
$\mathrm{HRPC}$ & 6 & 4 & 12 & 8 & $80.00 \% \mathrm{c}$ \\
\hline
\end{tabular}

a: comparison between BPH and PCa groups; b: comparison between PCa and HRPC groups; c: comparison between BPH and HRPC groups.

\subsection{Detection of GSTP1 Contents in Serum Samples by ELISA}

Difference was found among the serum concentrations of the $\mathrm{BPH}$ group, the PCa group and the HRPC group. The HRPC group showed serum GSTP1 concentration higher than that of the PCa group $(\mathrm{P}<0.01)$, the $\mathrm{BPH}$ group also showed serum GSTP1 concentration higher than that of the PCa group $(\mathrm{P}<0.01)$, and the difference between the serum concentrations of the BPH group and the HRPC group was not statistically significant $(\mathrm{P}>0.05)$ (Table 3$)$.

Table 3. Detection of GSTP1 concentrations in serum samples by ELISA ( $p s / m L)$.

\begin{tabular}{llll}
\hline Sample & BPH & PCa & HRPC \\
\hline GSTP1 concentration & $3607.2 \pm 136.8 \mathrm{a}$ & $1475.7 \pm 153.9 \mathrm{~b}$ & $2974.0 \pm 131.6 \mathrm{c}$ \\
P value & $\mathrm{P}<0.01$ & $\mathrm{P}<0.01$ & $\mathrm{P}>0.05$ \\
\hline
\end{tabular}

a: comparison between BPH and PCa groups; b: comparison between PCa and HRPC groups; c: comparison between BPH and HRPC groups.

\section{4. shRNA Screening Results}

RPM1640 culture solution with $10 \%$ fetal bovine serum was used. Place at $37^{\circ} \mathrm{C}$ in a $10 \% \mathrm{CO}_{2}$ incubator. When the DU145 cell fusion reached $80 \%$, they were subject to digestive passage with $0.5 \%$ trypsin and then inoculated (cell density of $1 \times 10^{5}$ ) to culture flasks or the orifice plates. They were used for experiment when the cell fusion reached $80 \%$ (Figure 2).

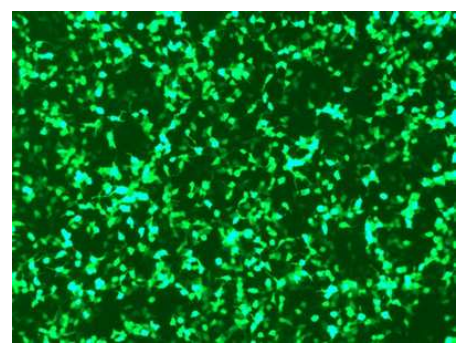

Figure 2. Morphology of DUl45 cells. 
The transfection efficiency levels of shRNA255, shRNA554 shRNA593 and Negative-shRNA of the GSTP1 gene were interfered. How to calculate the transfection efficiency: count the number of fluorescent cells on the fluorescence microscopic image; transfection efficiency $=$ number of fluorescent cells / total number of cells $\times 100 \%$. The requirement for transfection intervention would have been met where the shRNA vector transfection efficiency was $80 \%$ or more. Downstream analysis and testing can be performed (Figure 3, Table 4).
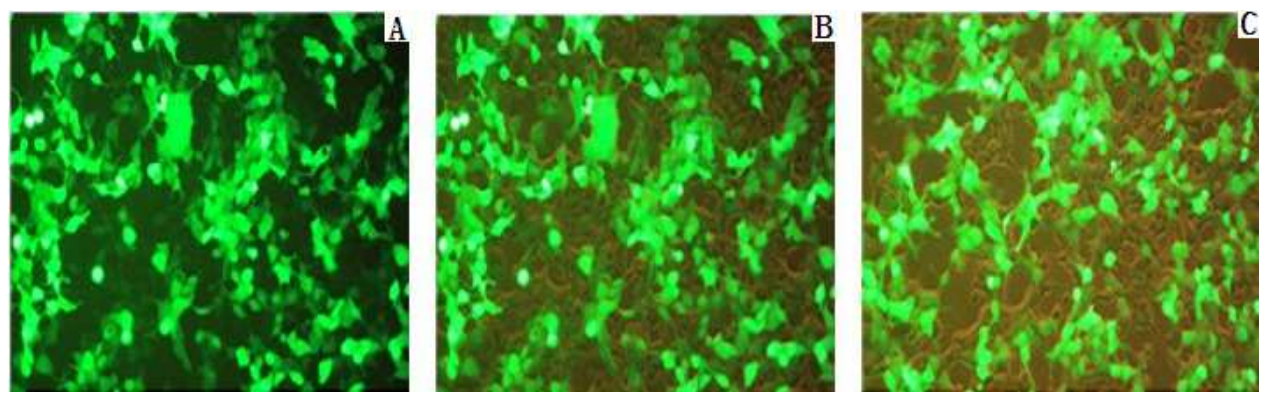

Figure 3. Transfected positive cells (fluorescence). A: GSTPl-shRNA255 transfected positive cells; B: GSTPl-shRNA554 transfected positive cells; C: GSTPl-shRNA593 transfected positive cells.

Table 4. shRNA transfection rates.

\begin{tabular}{llll}
\hline & shRNA255 & shRNA554 & shRNA593 \\
\hline Transfection rate & $52.78 \pm 3.65 \mathrm{a}$ & $68.51 \pm 2.97 \mathrm{~b}$ & $77.59 \pm 3.05 \mathrm{c}$ \\
$\mathrm{P}$ value & $\mathrm{P}<0.01$ & $\mathrm{P}<0.01$ & $\mathrm{P}<0.01$ \\
\hline
\end{tabular}

a: comparison between shRNA255 and shRNA554; b: comparison between shRNA554 and shRNA593; c: comparison between shRNA593 and shRNA255; d: comparison between shRNA554 and Nesative-shSNA.

\subsection{GSTP1 Gene mRNA Levels After Transfecting DU145 Cells with shRNA}

The transfection screening was divided into five groups: (1) DU145 cells + shRNA255; (2) DU145 cells + shRNA554; (3) DU145 cells + shRNA593; (4) DU145 cells + Negative-shRNA as a negative control vector group; and (5) DU145 cells + transfection reagent as a blank control group. After the separate transfection, the method the same as for testing the transfection efficiency was used to test the mRNA level of the DU145 cell GSTP1 genes after the transfection. They were divided into positive bands and analyzed with the Gel pro4.0 gel optical density analysis software to determine the IOD values.

The GSTP1 mRNA content decreased significantly after shRNA554 intervention, with an IOD value of $67.1 \pm 8.7$. The intervention effect was evidently lower than that of the carrier shRNA255 group $(162.2 \pm 12.6)$ and the shRNA593 group $(114.5 \pm 10.6)$ with statistically significant difference $(\mathrm{P}<0.01)$. No significant difference was found among the GAPDH reference genes of different groups $(\mathrm{P}>0.05)$. The content of GSTP1 mRNA decreased significantly, suggesting intervention effect significantly better than other carriers (Figure 4, Tables 5,6).

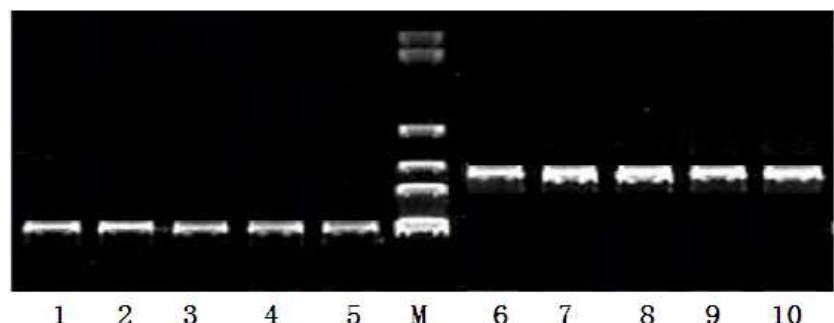

Figure 4. RT-PCR results of GSTP1 gene $m R N A$ levels after transfecting DU145 cells with shRNA. GSTP1 products (1 5): 237 bp, 1, 2, 3, 4 and 5 lanes are shRNA255, shRNA554, shRNA593, Negative-shRNA and blank control; GAPDH (6 10): $628 \mathrm{bp}, 6,7,0,9$ and 10 lanes are shRNA255, shRNA554, thRNA593, NegativHhRNA and blank control.

Table 5. GSTP1 gene mRNA contents after shRNA transfection.

\begin{tabular}{|c|c|c|c|c|c|}
\hline & 1 & 2 & 3 & 4 & 5 \\
\hline IOD value & $162.2 \pm 12.6 \mathrm{a}$ & $67.1 \pm 8.7 b$ & $114.5 \pm 10.6 \mathrm{c}$ & $239.1 \pm 11.7 d$ & $295.0 \pm 12.4 \mathrm{e}$ \\
\hline$P$ value & $\mathrm{P}<0.01$ & $\mathrm{P}<0.01$ & $\mathrm{P}<0.01$ & $\mathrm{P}<0.01$ & $\mathrm{P}<0.01$ \\
\hline
\end{tabular}

1 5: shRNA255, shRNA554, shRNA593, Negative-shRNA and blank control. a: comparison between shRNA255 and shRNA554; b: comparison between shRNA554 and shRNA593; c: comparison between shRNA593 and shRNA255; d: comparison between shRNA554 and Negative-shRNA; e: comparison between shRNA554 and blank control.

Table 6. GAPDH gene mRNA contents after shRNA transfection.

\begin{tabular}{llllll}
\hline & $\mathbf{6}$ & $\mathbf{7}$ & $\mathbf{8}$ & $\mathbf{9}$ & \\
\hline IOD value & $469.2 \pm 14.5 \mathrm{a}$ & $472.6 \pm 13.5 \mathrm{~b}$ & $464.9 \pm 14.2 \mathrm{c}$ & $470.5 \pm 13.8 \mathrm{~d}$ & $459.3 \pm 13.8 \mathrm{e}$ \\
$\mathrm{P}$ value & $\mathrm{P}>0.05$ & $\mathrm{P}>0.05$ & $\mathrm{P}>0.05$ & $\mathrm{P}>0.05$ & $\mathrm{P}>0.05$ \\
\hline
\end{tabular}

6 10: shRNA255, shRNA554, shRNA593, Negative-shRNA and blank control. a: comparison between shRNA255 and shRNA554; b: comparison between shRNA554 and shRNA593; c: comparison between shRNA593 and shRNA255; d: comparison between shRNA554 and Negative-shRNA; e: comparison between shRNA554 and blank control. 


\section{Discussion}

Recent studies on the relationship between the GSTP1 with prostate cancer mostly focused on the relationship between GSTP1 promoter methylation and the prostate cancer, including that between the GSTP1 promoter methylation and the clinical and pathological staging, Gleason scoring, recurrence rate and survival rate of the prostate cancer [9]. Yoon et al. compared tissues with prostatic hyperplasia and tissues with prostate cancer, and found that the GSTP1 gene promoter methylation in the tissues with prostate cancer was significantly higher than that in the tissues with prostatic hyperplasia. Besides, the GSTP1 methylation level of the prostate cancer cell strains was higher than that of the prostatic hyperplasia. Nevertheless, there was no clear correlation found between the GSTP1 methylation level and the PSA level, TNM staging, Gleason scoring and other clinical and pathological features of patients with prostate cancer [10]. Some studies suggest that gene promoter methylation is not the only reason for GSTP1 reduction in cancer tissues [11]. Clinical HRPC patients are often complicated with transdifferentiation of prostatic cancer, and further investigation is still needed to determine whether this is the cause of elevated GSTP1 levels. As to the relationship between the GSTP1 contents in the serum and tumor tissues, some researchers reported consistent GSTP1 contents in the endoscopic upper gastrointestinal tumor specimens and in its serum, both contents higher than that of the normal population. Besides, GSTP1 concentration was found significantly higher in patients with esophageal and gastric cancers than in the normal population. GSTP1 had increased expression in malignant tissues, but its protein activity in the malignant tissues was similar to that in the normal tissues [12]. In addition, change in GSTP1 genotype can also lead to progression of HRPC [13]. Through immunohistochemical examination, this experiment found that GSTP1 expressions in both the cancer tissues and serum of the HRPC subjects were higher than the early localized prostate cancer.

There has been little report about the cause of expression recovery by the GSTP1 in HRPC. The study conducted by Hauptstock et al. found that GSTP1 gene is expressed through activated fibroblasts or myofibroblasts. It can be inferred that during the process when the prostate cancer progresses to be androgen-independent, the GSTP1 expression recovered due to the stress response to the damage by the interstitial tissue may have led to the drug resistance in the HRPC [14]. Syeed found high expression in various multi-drug resistance genes including GSTP1, Bcl-2, topoisomerase II alpha and Ki-67 in the cancer tissues of the HRPC subjects after they were subject to transurethral resection of the prostate (TURP) that can relieve urination problems. High expression of these drug resistance genes was positively correlated with the Gleason scoring [15]. Therefore, the treatment targeting multidrug resistance genes can help to improve the drug sensitivity of cancer cells, thereby seeking a more effective therapy for HRPC.
GST- $\pi$ as a product of the GSTP1 gene encoding can serve the purpose of detoxification mainly by combining catalytically reduced glutathione with various electrophilic compounds to enhance the polarity of the glutathione and to promote the excretion of the environmental carcinogens [16]. GST- $\pi$ is an acidic protein that comprises two subunits with each subunit consisting of 209 amino acids. The high expression of GSTP1 in various malignant tumor tissues is highly related to the cancerization and tumor drug resistance [17]. More than $90 \%$ of the active GSTs in many tumor tissues are GSTP1, thus the relationship between GSTP1 and tumor has won widespread attention. As an important member of the glutathione S-transferase (GST) phase II metabolic enzyme family, GST- $\pi$ is capable of catalyzing the combination of glutathione and anticancer drugs and facilitating the discharge of these drugs. It has been proved by the latest studies that GST- $\pi$ can affect cell growth and apoptosis by recovering the protein-protein interactions and regulating the mitogen-activated protein kinase (MAPK) signaling passage [18]. High expression of GST- $\pi$ can cause drug resistance of tumor against cisplatin, doxorubicin, and other chemotherapeutic drugs, and suggests that GST- $\pi$ plays an important role in the primary and secondary drug resistance of tumor. To cope with the drug resistance mechanism of GST- $\pi$, there have been various clinical targeted therapies such as GST- $\pi$ inhibitor - glutathione analogue TER199, glutathione S-transferase inhibitor, etc. [19]. Some of them which have been put into pilot application in the clinical treatment enhanced the cytotoxic effects of chemotherapeutic drugs. In addition, RNA interference can radically inhibit the expression of the GSTP1 gene protein, which is expected to become the future trend of development of targeted therapy [20].

Most GST substrates are xenobiotics and oxidative stress products including environmental carcinogenic factors, which are used by the GSTs to promote the metabolism of carcinogenic factors and play an important role in damage resistance and anti-cancerization capacity of the cells [21]. GSTP1 is involved in the metabolism, detoxification and elimination of potential foreign complexes with genotoxicity. It can metabolize a variety of carcinogenic compounds, prevent DNA damage to the cells and formation of cancer cells, and suppress the DNA damage sensitivity increase and the occurrence of cancerization due to the GSTP1 activity [22]. The current studies suggest that there are several mechanisms for the drug resistance of tumor cells, including membrane glycoprotein-mediated drug efflux, drug metabolic disturbance mechanism; multidrug resistance associated protein (MRP), MDR caused by overexpression of lung resistance-related protein (LRP); enzyme mediation mechanism of MDR; apoptosis regulation gene-mediated DNA repair mechanism disturbance, DNA polymerase activity change mechanism; glutathione or glutathione S-transferase overexpression; protein kinase mechanism and other mechanisms [23]. Some studies believe that hypermethylation in the prostate cancer GSTP1 gene 
promoter region can lead to silent gene expression. This may occur in the early stage of the prostate cancer when decline in GSTP1 level leads to cancer progression. GSTP1 expression is elevated to promote the growth of the tumor after the prostate cancer progresses into the androgen-independent stage [24].

\section{Conclusion}

Based on the current studies, GSTP1 is expected to become a new tumor biomarker for the prostate cancer. Studies on gastrointestinal cancer show that GSTP1 is involved in the formation of drug resistance of tumor cells, and inhibition of GSTP1 activity can reduce the drug resistance of tumor cells and induce their apoptosis.

\section{References}

[1] Chung SD. Liu SP. et al. Association between Prostate Cancer and Urinary Calculi: A Population-Based Study [J]. PLoS One, 2019, 8 (2): 743-6.

[2] Mo Z. Gao Y. et al. An updating meta-analysis of the GSTM1, GSTT1, and GSTP1 polymorphisms and prostate cancer: a HuGE review [J]. Prostate, 2009, 69 (6): 662-88.

[3] Weroha SJ. Quevedo JF. et al. Treatment of hormone-refractory prostate cancer, HRPC, remains challenging [J]. Med Oncol, 2016, 27 (2): 569-71.

[4] Malakar M. Devi KR. et al. Genetic polymorphism of glutathione S-transferases M1 and T1, tobacco habits and risk of stomach cancer in Mizoram, India [J]. Asian Pac J Cancer Prev, 2018, 13 (9): 4725-32.

[5] Luo W. Kinsey M. et al. Glutathione s-transferases in pediatric cancer [J]. Front Oncol, 2017, 1 (39): 249-52.

[6] Jin P. Xie J. et al. Effect of gene GSTP1 silencing via shRNA transfection on androgen independent prostate cancer cell line Du145 [J]. Zhong Nan Da Xue Xue Bao Yi Xue Ban, 2018, 37 (8): 807-16.

[7] Gong M. Dong W. et al. Genetic polymorphisms of GSTM1, GSTT1, and GSTP1 with prostate cancer risk: a meta-analysis of 57 studies [J]. PLoS One, 2018, 7 (11): 587-9.

[8] Leite KR. Srougi M. et al. The use of immunohistochemistry for diagnosis of prostate cancer [J]. Int Braz J Urol, 2016, 36 (5): 583-90.

[9] Chiam K. Centenera MM. et al. GSTP1 DNA methylation and expression status is indicative of 5-aza-2'-deoxycytidine efficacy in human prostate cancer cells [J]. PLoS One, 2017, 6 (9): 634-6.

[10] Yoon HY. Kim SK. et al. Combined hypermethylation of APC and GSTP1 as a molecular marker for prostate cancer: quantitative pyrosequencing analysis $[\mathrm{J}]$. J Biomol Screen, 2018, 17 (7): 987-92.
[11] Qadri Q. Sameer AS. et al. Genetic polymorphism of the glutathione-S-transferase P1 gene (GSTP1) and susceptibility to prostate cancer in the Kashmiri population [J]. Genet Mol Res, 2017, 10 (4): 3038-45.

[12] Zhao Y. Wang F. et al. Genetic polymorphism of p53, but not GSTP1, is association with susceptibility to esophageal cancer risk - a meta-analysis [J]. Int J Med Sci, 2016, 7 (5): 300-8.

[13] Re A. Aiello A. et al. Silencing of GSTP1, a prostate cancer prognostic gene, by the estrogen receptor-beta and endothelial nitric oxide synthase complex [J]. Mol Endocrinol, 2017, 25 (12): 2003-16.

[14] Hauptstock V. Kuriakose S. et al. Glutathione-S-transferase pi 1 (GSTP1) gene silencing in prostate cancer cells is reversed by the histone deacetylase inhibitor depsipeptide [J]. Biochem Biophys Res Commun, 2017, 412 (4): 606-11.

[15] Syeed N. Syed Sameer A. et al. Promoter methylation profile of GSTP1 and RASSF1A in benign hyperplasia and metastatic prostate cancer patients in a Kashmiri population [J]. Mol Med Rep, 2017, 3 (5): 883-7.

[16] Kiran B. Karkucak M. et al. GST (GSTM1, GSTT1, and GSTP1) polymorphisms in the genetic susceptibility of Turkish patients to cervical cancer [J]. J Gynecol Oncol, 2016, 21 (3): $169-73$.

[17] Wang J. Zhang J. et al. Expression of P-gp, MRP, LRP, GST-pi and TopoIIalpha and intrinsic resistance in human lung cancer cell lines [J]. Oncol Rep, 2017, 26 (5): 1081-9.

[18] Lu D. Shi HC. et al. Multidrug resistance-associated biomarkers PGP, GST-pi, Topo-II and LRP as prognostic factors in primary ovarian carcinoma $[\mathrm{J}]$. Br J Biomed Sci, 2017, 68 (2): 69-74.

[19] Zhang Y. Liu J. et al. Transgenic alfalfa plants co-expressing glutathione S-transferase (GST) and human CYP2E1 show enhanced resistance to mixed contaminates of heavy metals and organic pollutants [J]. J Hazard Mater, 2017, 189 (2): 357-62.

[20] Hasegawa K. Miwa S. et al. Allyl isothiocyanate that induces GST and UGT expression confers oxidative stress resistance on C. elegans, as demonstrated by nematode biosensor [J]. PLoS One, 2016, 5 (2): 267-9.

[21] Bai YL. Zhou B. et al. Predictive role of GSTs on the prognosis of breast cancer patients with neoadjuvant chemotherapy [J]. Asian Pac J Cancer Prev, 2018, 13 (10): 5019-22.

[22] Richiardi L. Fiano V. et al. Promoter methylation in APC, RUNX3, and GSTP1 and mortality in prostate cancer patients [J]. J Clin Oncol, 2009, 27 (19): 3161-8.

[23] Pandey M. Shukla S. et al. Promoter demethylation and chromatin remodeling by green tea polyphenols leads to re-expression of GSTP1 in human prostate cancer cells [J]. Int J Cancer, 2016, 126 (11): 2520-33.

[24] Vardi A. Bosviel R. et al. Soy phytoestrogens modify DNA methylation of GSTP1, RASSF1A, EPH2 and BRCA1 promoter in prostate cancer cells [J]. In Vivo, 2016, 24 (4): 393-400. 